\title{
Adjuvant chemotherapy following concurrent chemoradiotherapy for uterine cervical cancer with lymphadenopathy
}

\author{
AKIKO ABE $^{1}$, HIROYUKI FURUMOTO ${ }^{1}$, MASATO NISHIMURA ${ }^{1}$, MINORU IRAHARA $^{1}$ and HITOSHI IKUSHIMA ${ }^{2}$ \\ ${ }^{1}$ Department of Obstetrics and Gynecology, Institute of Health Biosciences, The University of Tokushima Graduate School; \\ ${ }^{2}$ Department of Radiology, Tokushima University School of Medicine, Tokushima 770-8503, Japan
}

Received July 8, 2011; Accepted October 14, 2011

DOI: $10.3892 / \mathrm{ol} .2011 .516$

\begin{abstract}
The present study aimed to retrospectively evaluate the efficacy and toxicities of adjuvant chemotherapy with paclitaxel and carboplatin (TC) following concurrent cisplatin-based chemoradiation (CCRT) in patients with cervical cancer with lymphadenopathy (N1). A total of 37 patients with FIGO stage IB2-IVA cervical carcinoma with N1 (median age 57 years, range 31-74 years) were enrolled. External beam radiation therapy was followed by high-dose-rate brachytherapy. In cases of suspected para-aortic lymphadenopathy or common iliac lymph node involvement, extended radiotherapy fields were applied. Positive lymph nodes were externally radiated. Cisplatin was administered weekly at a dose of $30 \mathrm{mg} / \mathrm{m}^{2}$ during external beam radiation therapy. Adjuvant therapy was administered to 17 patients and comprised carboplatin $(6 \mathrm{mg} / \mathrm{ml} / \mathrm{min})$ and paclitaxel $\left(175 \mathrm{mg} / \mathrm{m}^{2}\right)$ administered monthly after CCRT, and repeated every 4 weeks for 3-6 cycles. Over a median 21.5-month follow-up, no significant differences were found in the recurrence rate, progression-free survival, overall survival, or median interval to recurrence with $\mathrm{N} 1$ cervical cancer patients between the two groups. Patients with para-aortic lymphadenopathy who received CCRT and adjuvant chemotherapy had a more favorable overall and disease-free survival than those treated with CCRT alone. However, 16/17 patients developed grade 3-4 leukopenia and 14/17 patients developed severe hematologic toxicity during adjuvant chemotherapy. In conclusion, adjuvant chemotherapy consisting of full dose TC therapy after CCRT was not well tolerated in general and exhibited no benefit to N1 cervical cancer patients. However, it may be of therapeutic advantage over CCRT alone in cervical cancer patients with para-aortic lymphadenopathy.
\end{abstract}

Correspondence to: Dr Akiko Abe, Department of Obstetrics and Gynecology, Institute of Health Biosciences, The University of Tokushima Graduate School, 3-18-15 Kuramoto-cho, Tokushima 770-8503, Japan

E-mail: akiko06090111@yahoo.co.jp

Key words: cervical cancer, lymphadenopathy, chemoradiation, adjuvant chemotherapy

\section{Introduction}

It is estimated that in 2005 in Japan, 8,474 women were diagnosed with cervical carcinoma and approximately 2,500 women succumbed to the disease (1). A National Cancer Institute clinical announcement based on 5 trials concluded that concurrent cisplatin-based chemoradiation (CCRT) improved survival in patients with advanced cervical carcinoma (2-6). CCRT includes a combination of external beam radiotherapy and intracavity brachytherapy. In Japan, CCRT is generally performed in patients with locally advanced disease and/or lymphadenopathy, who are at high risk for recurrence.

Green et al showed that an absolute survival benefit of $12 \%$ was potentially attributable to the use of chemoradiotherapy (7). The Radiation Therapy Oncology Group trial (RTOG) 90-01 showed after long-term follow-up that CCRT did not decrease para-aortic recurrence, and that more than $50 \%$ of patients with para-aortic recurrence revealed distant metastases after CCRT (6). No standard treatment exists for cervical cancer with lymphadenopathy after CCRT. However, studies pertaining to adjuvant chemotherapy or consolidation chemotherapy after CCRT in cases of cervical cancer with lymphadenopathy are available. Kim et al (8) compared treatment with CCRT followed by adjuvant chemotherapy to treatment with CCRT alone in FIGO stage IB and IIB bulky cervical cancers. The chemotherapeutic agents they used comprised platinum followed by 5 consecutive daily infusions of 5-fluorouracil every 3 weeks, and resulted in no therapeutic advantage (8). However, a phase II study showed that consolidation chemotherapy consisting of cisplatin and 5-fluorouracil every 3 weeks after CCRT was tolerable and effective (9).

The present study retrospectively evaluated the efficacy and toxicities of adjuvant chemotherapy with paclitaxel and carboplatin followed by CCRT for patients with cervical cancer with lymphadenopathy.

\section{Materials and methods}

Patient eligibility. Patients with FIGO Stage IB2-IVA cervical carcinoma with lymphadenopathy who were treated with CCRT at Tokushima University Hospital, Japan, were enrolled. Patients who had received chemotherapy or radiotherapy prior to this study were excluded. Eligibility criteria included: age $<75$ years, Eastern Cooperation Oncology 
Group (ECOG) performance status score of $0-1$, normal cardiovascular function, normal blood cell counts, and normal serum levels of blood urea nitrogen, creatinine, and bilirubin. Criteria for malignancy determined by positron emission tomography/computed tomography (PET/CT) or magnetic resonance imaging (MRI) were a pelvic or para-aortic lymph node with a short-axis dimension of $\geq 1 \mathrm{~cm}$ or a standard uptake value (SUV) of $\geq 3$. Each patient provided written informed consent prior to enrollment.

Patient characteristics. Between September 2005 and December 2009, 37 patients with FIGO stage IB2-IVA cervical cancer who had pelvic and/or para-aortic lymphadenopathy were treated with CCRT with or without adjuvant chemotherapy. Patient characteristics are shown in Table I. The median age was 57 years (range 31-74). Distribution of patients by stage was: IB2, $n=3$; II, $n=17$; III, $n=13$; and IVA, $n=4$. Histology revealed squamous cell carcinoma in 33 patients (89\%) and other cell types in 4 patients (11\%). Characteristics of patients in the CCRT-alone and CCRT plus adjuvant chemotherapy (CCRT-CTX) groups are shown in Table I. The CCRT-alone group comprised 20 patients, whereas the CCRT-CTX group comprised 17 patients. A total of 15 patients had para-aortic lymph node enlargement $>10 \mathrm{~mm}$ on the minimum diameter as assessed by PET-CT or MRI. In the CCRT alone group, 4 patients had para-aortic lymphadenopathy and 11 patients had involvement of $\geq 3$ pelvic lymph nodes. In the CCRT-CTX group, 11 patients had para-aortic lymphadenopathy and 12 patients had involvement of $\geq 3$ pelvic lymph nodes.

Radiotherapy. All 37 patients underwent radiotherapy (RT) with a combination of external beam RT (EBRT) and intracavitary brachytherapy according to the general rules for clinical and pathological management of uterine cervical cancer (10). EBRT consisting of whole pelvic RT (WPRT) and center-shielding WPRT was delivered using a 10-MV photon by a linear accelerator (PRIMUS High-Energy; Toshiba Medical Systems Co., Tochigi, Japan) with each patient in the supine position. Gross tumor volume (GTV) was defined as the primary tumor and involved lymph nodes. Clinical target volume (CTV) encompassed the GTV with $0.5-\mathrm{cm}$ isotropic margin and uterus, presacral, common iliac, internal iliac, upper external iliac, and obturator lymph node. Planning target volume (PTV) was defined as the CTV with an isotropic margin of $1 \mathrm{~cm}$. A total of 5 weekly fractions of 2.0 Gy per fraction, at a total dose of 20-40 Gy in 10-20 fractions, were delivered to the isocenter with WPRT using a four-field box technique. The dose distributions were computed using the Convolution Algorithm implemented in the Xio planning system (CMS Inc., St. Louis, MO, USA). These fields realized dose delivery to the PTV of not $<95 \%$ of the prescription dose. An additional 10-30 Gy in 5-15 fractions was delivered with center-shielding WPRT using anteroposterior and posteroanterior parallel-opposing fields after WPRT at a total dose of $50 \mathrm{~Gy}$ in 25 fractions. High-dose-rate (HDR) intracavitary brachytherapy using a high-activity Ir-192 source was started 7-21 days after the initiation of EBRT. HDR intracavitary brachytherapy and EBRT were not administered on the same day. A total dose of 15 Gy in 3 fractions or
Table I. Patient characteristics $(\mathrm{n}=37)$.

\begin{tabular}{|c|c|c|c|}
\hline Characteristics & $\begin{array}{l}\text { CCRT-alone } \\
\text { group }\end{array}$ & $\begin{array}{l}\text { CCRT-CTX } \\
\text { group }\end{array}$ & p-value \\
\hline Number of patients & 20 & 17 & \\
\hline $\begin{array}{l}\text { Median age } \\
\text { (years) (range) }\end{array}$ & $57(31-72)$ & $53(32-72)$ & \\
\hline \multicolumn{4}{|l|}{ FIGO stage } \\
\hline IB & 2 & 1 & NS \\
\hline IIA & 1 & 0 & \\
\hline IIB & 9 & 7 & \\
\hline IIIA & 1 & 1 & \\
\hline IIIB & 5 & 6 & \\
\hline IVA & 2 & 2 & \\
\hline \multicolumn{4}{|l|}{ Histology } \\
\hline $\begin{array}{l}\text { Squamous cell } \\
\text { carcinoma }\end{array}$ & 20 & 13 & 0.036 \\
\hline $\begin{array}{l}\text { Non-squamous cell } \\
\text { carcinoma }\end{array}$ & 0 & 4 & \\
\hline \multicolumn{4}{|l|}{$\begin{array}{l}\text { No. of pelvic lymph } \\
\text { node involvement }\end{array}$} \\
\hline 1 & 4 & 2 & NS \\
\hline 2 & 5 & 3 & \\
\hline 3 & 2 & 5 & \\
\hline$\geq 4$ & 9 & 7 & \\
\hline \multicolumn{4}{|c|}{$\begin{array}{l}\text { Para-aortic lymph node } \\
\text { involvement }\end{array}$} \\
\hline Positive & 4 & 11 & \\
\hline Negative & 16 & 6 & 0.007 \\
\hline \multicolumn{4}{|l|}{$\begin{array}{l}\text { CCRT: Number of } \\
\text { CDDP cycles }\end{array}$} \\
\hline 1 & 0 & 1 & NS \\
\hline 2 & 2 & 1 & \\
\hline 3 & 3 & 3 & \\
\hline 4 & 6 & 3 & \\
\hline 5 & 8 & 10 & \\
\hline
\end{tabular}

CCRT, concurrent chemoradiotherapy; CTX, chemotherapy; NS, not significant; CDDP, cisplatin.

24 Gy in 4 fractions at Point A was delivered by weekly HDR brachytherapy. When para-aortic lymph node or common iliac lymph node involvement was suspected, patients were treated with extended EBRT fields including para-aortic lymph nodes using anteroposterior and postroanterior parallel-opposing portals. The superior border of the para-aortic field was a transverse line through the T12-L1 intervertebral space. The total dose delivered to the whole pelvis and para-aortic lymph node was $45 \mathrm{~Gy}$ adminsitered at a dose of 1.8 Gy per fraction. Concomitant boost of 6-10 Gy in 3-5 fractions was added to the metastatic lymph nodes in all clinically node-positive patients.

Chemotherapy. The protocol for concurrent chemotherapy was based on a previous report, and consisted of cisplatin 
Table II. Characteristics of patients in the CCRT-alone and CCRT plus adjuvant chemotherapy groups.

A, Acute systemic toxicity during CCRT.

\begin{tabular}{lcc}
\hline & $\begin{array}{c}\text { Non-extended field } \\
\mathrm{n}=18(\%)\end{array}$ & $\begin{array}{c}\text { Extended field } \\
\mathrm{n}=19(\%)\end{array}$ \\
\hline $\begin{array}{l}\text { ( cycles of CDDP } \\
\text { Number of patients with } \\
\text { grade 3-4 acute toxicity }\end{array}$ & $14(78)$ & $13(68)$ \\
Hematologic toxicity & & $12(63)$ \\
Leukopenia & $8(44)$ & $11(58)$ \\
Thrombocytopenia & $7(38)$ & $0(0)$ \\
Anemia & $0(0)$ & $3(16)$ \\
Gastrointestinal toxicity & $1(5.3)$ & $4(22)$ \\
\hline
\end{tabular}

B, Acute systemic toxicity during adjuvant chemotherapy.

\begin{tabular}{lcc}
\hline & $\begin{array}{c}\text { Non-extended field } \\
\mathrm{n}=4(\%)\end{array}$ & $\begin{array}{c}\text { Extended field } \\
\mathrm{n}=13(\%)\end{array}$ \\
\hline $\begin{array}{l}\text { Number of patients } \\
\text { with grade 3-4 acute }\end{array}$ & $4(100)$ & $13(100)$ \\
hematologic toxicity & & \\
Leukopenia & $4(100)$ & $12(92)$ \\
Thrombocytopenia & $0(0)$ & $3(23)$ \\
Anemia & $0(0)$ & $7(54)$ \\
\hline
\end{tabular}

C, Late treatment-related toxicities.

\begin{tabular}{lcccc}
\hline & \multicolumn{2}{c}{ CCRT-alone (n=20) } & & CCRT-CTX (n=17) \\
\cline { 2 - 3 } & Grade 1-2 & Grade 3-5 & & Grade 1-2 \\
\hline Site of effect & 3 & 1 & 2 & \\
Gastrointestinal & 0 & 0 & 1 & 0 \\
Genitourinary & 1 & 0 & 2 & 0 \\
Insufficiency fractures & & & 2 \\
\hline
\end{tabular}

administered weekly at a dose of $30 \mathrm{mg} / \mathrm{m}^{2}$ during EBRT (11). Granisetron ( $3 \mathrm{mg}$ ) was routinely administered as an anti-emetic treatment, and $8 \mathrm{mg}$ of dexamethasone was received by patients who complained of severe nausea. Cisplatin was withheld if grade $\geq 3$ gastrointestinal toxicities appeared, or when either the leukocyte count was $<3,000 / \mathrm{mm}^{3}$ or the platelet count was $<100,000 / \mathrm{mm}^{3}$.

Adjuvant chemotherapy. TC therapy [carboplatin $(\mathrm{AUC}=6)$ and paclitaxel $\left.\left(175 \mathrm{mg} / \mathrm{m}^{2}\right)\right]$ was administered monthly following CCRT. Paclitaxel was administered as a 3-h intravenous infusion and carboplatin as a 2-h intravenous infusion. Chemotherapy was repeated every 4 weeks for 3-6 cycles. Toxicity of the regimen was determined according to the Common Terminology Criteria for Adverse Events (CTCAE) version 4.0. In cases of severe toxicity (grade $\geq 3$ ), chemotherapy was postponed until toxic symptoms disappeared. Chemotherapy was interrupted when the leukocyte count was $<3,000 / \mathrm{mm}^{3}$, platelet count was $<100,000 / \mathrm{mm}^{3}$, hemoglobin was $<80 \mathrm{~g} / 1$, or grade $\geq 3$ non-hematologic toxicity developed.

Study endpoint. The primary endpoint was acute toxicity. Acute toxicity was assessed according to the National Cancer Institute-Common Toxicity Criteria (NCI-CTC). Secondary endpoints were treatment failures, survival rates, disease-free survival rate, and late toxicity. Late toxicity for RT was assessed according to the RTOG criteria. Overall survival (OS) was calculated from the time of initiation of therapy to the time of death or last visit. Progression-free survival (PFS) was measured from the initiation of therapy to the date of last visit or the radiographic evidence of progressive disease.

Statistical analysis. Treatment outcomes were compared between the CCRT-alone and CCRT-CTX groups retrospectively. Survival curves were estimated by the Kaplan-Meier method and compared using the log-rank test. The differences between the two groups according to treatment 
assignment and patient characteristics were assessed using the Chi-square analysis.

\section{Results}

Toxicity. A total of 151 cycles of CCRT and 78 cycles of adjuvant chemotherapy were administered. Table II shows the patients who developed acute toxicity. A total of 27 patients (73\%) received $\geq 4$ cycles of cisplatin chemotherapy (CDDP) during RT. In the remaining 4 patients, CDDP was interrupted by gastrointestinal toxicity in 3 patients, and by failure of renal function in 1 patient. None of the patients succumbed to their disease during treatment. During CCRT, grade 3 leukopenia accounted for $48.6 \%$ (18 patients) of toxicity cases. Grade 3-4 anemia occurred in 4 patients $(11 \%)$. No patients suffered from thrombocytopenia during CCRT. A total of 19 patients were treated with extended field radiotherapy. Table IIA shows the toxicity with or without para-aortic irradiation. A total of 12 patients $(63 \%)$ who were treated with extended field irradiation developed grade 3-4 hematologic toxicity. No significant difference was observed in grade 3-4 hematologic toxicity with or without para-aortic irradiation during CCRT $(\mathrm{p}=0.121)$. Grade 3 gastrointestinal toxicity during CCRT occurred in 5 patients $(13 \%)$.

Adjuvant chemotherapy following CCRT and complications of treatment. Adjuvant CT consisted of 3-6 cycles of TC therapy after CCRT. Only 1 patient was treated with weekly TC therapy due to severe hematologic toxicity during CCRT, and 2 patients were treated with docetaxel and carboplatin (DC) therapy due to numbness. The median interval of CCRT and adjuvant chemotherapy was 44 days. The median inter-cycle intervals of chemotherapy were $28 \pm 8.7,28 \pm 7.1,35 \pm 14.5,28 \pm 6.7$ and $28 \pm 9.3$ days. Table IIB shows that during adjuvant chemotherapy grade 3-4 leukopenia occurred in almost all of the patients (16/17 patients). Grade 3 or higher anemia and thrombocytopenia occurred in 7 and 3 patients, respectively. Severe hematologic toxicity in 14 patients $(82 \%)$ resulted in a dose reduction of the agents by $20 \%$. Due to prolonged hematologic toxicities, 2 patients discontinued chemotherapy. Chemotherapy schedules were delayed and dose-reduced in 14 patients $(82 \%)$ and 2 patients $(5 \%)$ discontinued adjuvant chemotherapy due to hematologic toxicity. Adjuvant chemotherapy comprising full-dose TC therapy following CCRT was not well tolerated in general.

Late complications of treatment are shown in Table IIC. A total of 9 patients $(24 \%)$ had grade 2 late complications. There were 2 incidences of grade 3 or 4 gastrointestinal toxicity, but no late treatment-related fatalities.

Treatment outcome. The median follow-up was 21.5 months (range 14.8-36). A total of 13 patients (35\%) had recurrence ( 8 in the CCRT-alone group, and 5 in the CCRT-CTX group). No significant differences were found in the recurrence rate, PFS $(\mathrm{p}=0.751)$ or OS $(\mathrm{p}=0.813)$ (Fig. 1). The median interval to recurrence was 6.7 months (range 4.3-22.) without adjuvant chemotherapy and 10.4 months (range 3.2-14.8) with adjuvant chemotherapy. In the CCRT-alone group, 8 patients had recurrence, and the recurrence sites were inside the radiation field in 6 patients and outside the radiation field in 3 patients
Table III. Pattern of recurrence.

A, Pattern of recurrence.

\begin{tabular}{lccc}
\hline & $\begin{array}{c}\text { CCRT-alone } \\
(\mathrm{n}=20)\end{array}$ & $\begin{array}{c}\text { CCRT-CTX } \\
(\mathrm{n}=17)\end{array}$ & p-value \\
\hline Recurrence & $8(40 \%)$ & $5(29 \%)$ & \\
Locoregional failure & $5(25 \%)$ & $2(12 \%)$ & $\mathrm{NS}$ \\
Lymph node failure & $4(20 \%)$ & $4(23 \%)$ & $\mathrm{NS}$ \\
$\begin{array}{l}\text { Distant metastasis } \\
\text { (excluding para-aortic }\end{array}$ & $2(10 \%)$ & $1(6 \%)$ & $\mathrm{NS}$ \\
failure) & & & \\
Inside radiation field & $6(30 \%)$ & $4(23 \%)$ & $\mathrm{NS}$ \\
Outside radiation field & $3(15 \%)$ & $2(12 \%)$ & $\mathrm{NS}$ \\
\hline
\end{tabular}

B, Pattern of recurrence of patients who underwent para-aortic lymphadenopathy $(n=15)$.

\begin{tabular}{lccc}
\hline & $\begin{array}{c}\text { CCRT-alone } \\
(\mathrm{n}=4)\end{array}$ & $\begin{array}{c}\text { CCRT-CTX } \\
(\mathrm{n}=11)\end{array}$ & p-value \\
\hline Recurrence & $3(75 \%)$ & $4(36 \%)$ & $\mathrm{NS}$ \\
Locoregional failure & 2 & 1 & $\mathrm{NS}$ \\
$\begin{array}{l}\text { Lymph node failure } \\
\begin{array}{l}\text { Distant metastasis } \\
\text { (excluding para-aortic }\end{array}\end{array}$ & 2 & 4 & $\mathrm{NS}$ \\
failure) & & 3 & $\mathrm{NS}$ \\
\end{tabular}

NS, not significant.

(Table III). In the CCRT-CTX group, 5 patients had recurrence, and the recurrence sites were inside the radiation field in 4 patients and outside the radiation field in 2 patients. The cumulative incidence of lymph node metastases was 20 and $23 \%$. The incidence of distant metastases, including paraaortic lymph node metastases, was not reduced by adjuvant chemotherapy. A total of 7 patients had locoregional recurrences: 5 pelvic recurrences without adjuvant chemotherapy and only 2 recurrences with adjuvant chemotherapy. No significant differences were observed in the patterns of failure between the two groups.

Para-aortic lymphadenopathy was demonstrated by imaging in 15 patients: 4 in the CCRT-alone group and 11 in the CCRT-CTX group. A total of 6 patients (40\%) with paraaortic lymphadenopathy had lymph node failure: 2 patients without adjuvant chemotherapy and 4 patients with adjuvant chemotherapy. In the CCRT-CTX group, the estimated 2-year PFS was $60.1 \%$ and OS was $90 \%$ (Fig. 2).

\section{Discussion}

Lymph node metastasis is one of the most significant prognostic factors of cervical cancer. According to the literature, the incidence of lymph node involvement increases with the FIGO stage: it occurs in $12-22 \%$ of stage IB, $10-27 \%$ of stage IIA, and 34-43\% of stage IIB (12). A GOG (Gynecology Oncology Group) study reported that the para-aortic lymph 
A

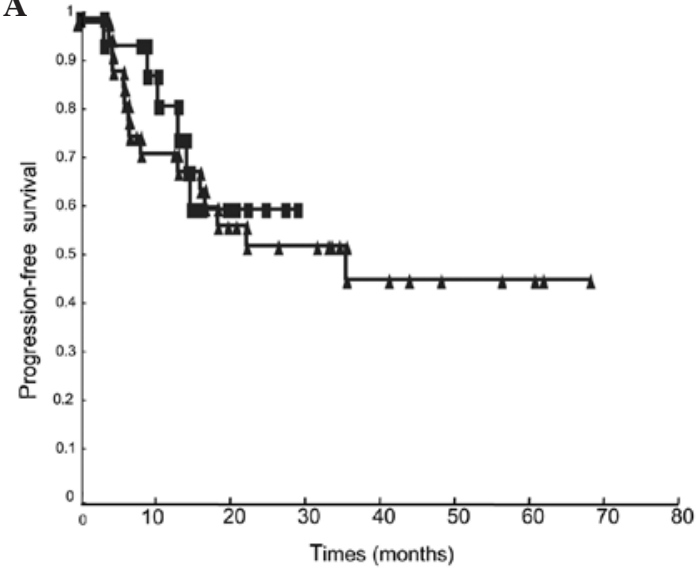

B

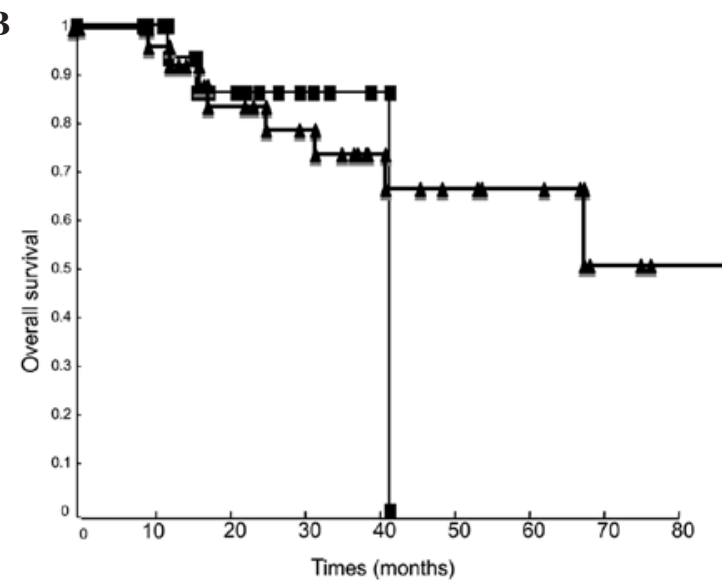

Figure 1. Survival curve among patients with lymphadenopathy in the CCRT-only and CCRT-CTX groups. (A) Progression free-survival ( $\mathrm{p}=0.751$ ).

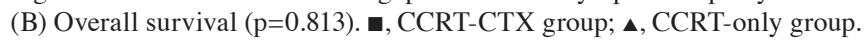

A

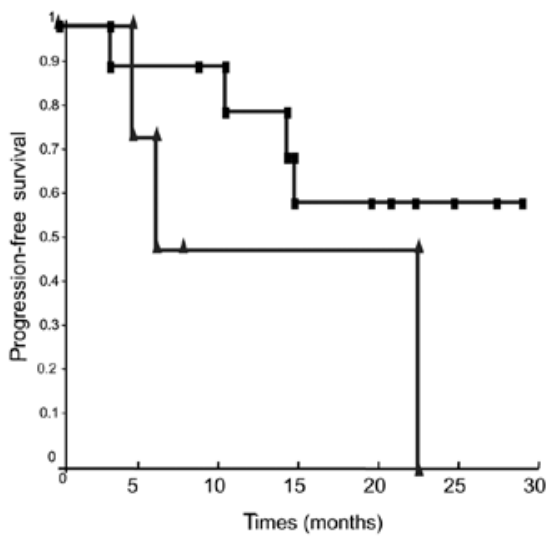

B

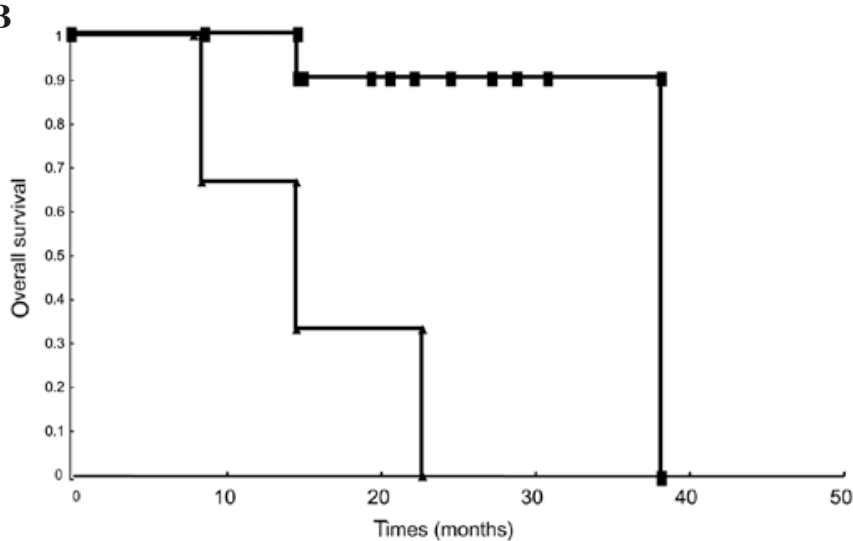

Figure 2. Survival curve among patients who had para-aortic lymphadenopathy in the CCRT-only and CCRT-CTX groups. (A) Progression-free survival $(\mathrm{p}=0.120)$. (B) Overall survival ( $\mathrm{p}=0.001)$. $\mathbf{n}$, CCRT-CTX group; $\mathbf{\Delta}$, CCRT-only group.

node metastasis in cervical cancer was $6 \%$ for stage I, $16 \%$ for stage II, and $25 \%$ for stage III (13). In the GOG study, the patients underwent surgical staging, and the 3-year survival for those with stage IIB and IIIB cancers was $25 \%$. Paraaortic lymph involvement was also a significant prognostic factor (14). Para-aortic node metastases occurred in $76 \%$ of patients with positive common iliac lymph nodes, and in $19 \%$ of patients without common iliac lymph node involvement. Common iliac and para-aortic lymph node metastases are associated with markedly poor survival.

Evidence for the use of CCRT has been established for patients without para-aortic node metastases through the pathological evaluation of lymph node status by surgical staging. In the present study, lymphadenectomy was not performed prior to CCRT, as is common in Japan. Treatment was planned by analysis of MRI or PET-CT, and not by surgical staging. Evaluation of lymph node involvement with noninvasive techniques such as MRI provides a sensitivity and specificity near $80 \%$ when nodes are $>1 \mathrm{~cm}$. Such percentages may decrease to $24-48 \%$ in cases of lymph nodes with small metastases $(14,15)$. Compared to the results observed with regards to $\mathrm{CT}$ alone, PET-CT may detect smaller para-aortic disease (16).
At least 7 trials have demonstrated an apparent improvement in local pelvic failures [19.3\% (223/1155) in a CCRT group versus $31.7 \%$ (313/986) in an RT-alone group]. CDDP in CCRT may have a synergistic effect on micrometastases (17). In addition, Green et al demonstrated a significant benefit of CCRT on local and distant recurrences (7). In the present study, $73 \%$ (33/37) of patients had $\geq 3$ cycles of CDDP, and $81 \%$ (30/37) of patients had no local pelvic failures.

For the purpose of modulating micrometastases and lymph node metastases, patients with pelvic or para-aortic lymph node involvement were treated with extended field radiotherapy. CCRT with extended field pelvic para-aortic irradiation has been reported $(18,19)$. A GOG study showed that radiotherapy with extended field was completely treated in $37 \%$ of patients at 8 weeks and $68 \%$ of patients at 9 weeks (19). TP therapy [paclitaxel $\left(40 \mathrm{mg} / \mathrm{m}^{2}\right)$ and cisplatin $\left(40 \mathrm{mg} / \mathrm{m}^{2}\right)$ ] with extended pelvic and para-aortic beam RT was acceptable. In the present study, grade $\geq 3$ acute hematologic toxicity was observed in $63 \%(12 / 19)$ of patients who received extended field radiation and CDDP $\left(30 \mathrm{mg} / \mathrm{m}^{2}\right)$ chemotherapy as CCRT. The regimen with pelvic and para-aortic lymph irradiation was tolerable.

In a prospective study, Choi et al reported that consolidation chemotherapy with cisplatin and 5-fluorouracil following 
CCRT was well tolerated and effective in patients with locally advanced cervical carcinoma (9). Zhang et al reported CCRT with paclitaxel $\left(135 \mathrm{mg} / \mathrm{m}^{2}\right)$ and nedaplatin $\left(60 \mathrm{mg} / \mathrm{m}^{2}\right)$ followed by consolidation chemotherapy with FIGO stage IIB-IIIB cervical cancer (20). These authors found that consolidation chemotherapy every 3 weeks for 4 cycles was effective and well tolerated: 31 patients $(91 \%)$ received more than 3 cycles of consolidation chemotherapy.

TP therapy has been reported to be highly effective in advanced and recurrent cervical cancer (21). TC therapy has been reported to be as effective as TP therapy (22). The chemotherapy comprising paclitaxel and a platinum agent has been evaluated, and in the present study, TC therapy after CCRT was administered as adjuvant chemotherapy and comprised paclitaxel $\left(175 \mathrm{mg} / \mathrm{m}^{2}\right)$ and carboplatin $(\mathrm{AUC}=6)$. Adjuvant chemotherapy following CCRT increased the severity of the hematologic toxicities, whereas the frequency of other toxicities was not different between the 2 groups. In the two groups, a total of $5 \%(2 / 37)$ of patients had grade $\geq 3$ late toxicities. In the RTOG 90-01 study, grade $\geq 3$ late gastrointestinal toxicities occurred $3-9 \%$ of the time over 5 years (6). In the present study, 3 more cycles of chemotherapy consisting of paclitaxel and carboplatin were administered following CCRT. Adjuvant chemotherapy schedules were either delayed or dose-reduced in 14 patients $(82 \%)$, and 2 of 37 patients $(5 \%)$ were unable to receive adjuvant chemotherapy due to toxicity. Therefore, due to the small number of patients, it is unclear as to whether the chemotherapy dose in this study was tolerable.

In conclusion, patients with para-aortic lymph node metastasis in stage IB2-IVA cervical cancer who received CCRT and adjuvant chemotherapy had a more favorable survival than those treated with CCRT alone. However, no advantage of adjuvant chemotherapy was observed in patients with pelvic and para-aortic lymphadenopathy. Future clinical trials are required for confirmation of the clinical efficacy of adjuvant chemotherapy.

\section{References}

1. Center for Cancer Control and Information Services, National Cancer Center, Japan, 2010 (ganjoho.jp).

2. Morris M, Eifel PJ, Lu J, Grigsby PW, Levenback C, Stevens RE, Rotman M, Gershenson DM and Mutch DG: Pelvic radiation with concurrent chemotherapy compared with pelvic and paraaortic radiation for high-risk cervical cancer. N Engl J Med 340: 1137-1143, 1999.

3. Rose PG, Bundy BN, Watkins EB, Thigpen JT, Deppe G, Maiman MA, Clarke-Pearson DL and Insalaco S: Concurrent cisplatin-based radiotherapy and chemotherapy for locally advanced cervical cancer. N Engl J Med 340: 1144-1153, 1999.

4. Whitney CW, Sause W, Bundy BN, Malfetano JH, Hannigan EV, Fowler WC Jr, Clarke-Pearson DL and Liao SY: Randomized comparison of fluorouracil plus cisplatin versus hydroxyurea as an adjunct to radiation therapy in stage IIB-IVA carcinoma of the cervix with negative para-aortic lymph nodes: a Gynecologic Oncology Group and Southwest Oncology Group study. J Clin Oncol 17: 1339-1348, 1999.
5. Keys HM, Bundy BN, Stehman FB, Muderspach LI, Chafe WE, Suggs CL III, Walker JL and Gersell D: Cisplatin, radiation, and adjuvant hysterectomy compared with radiation and adjuvant hysterectomy for bulky stage IB cervical carcinoma. N Engl J Med 340: 1154-1161, 1999.

6. Eifel PJ, Winter K, Morris M, Levenback C, Grigsby PW, Cooper J, Rotman M, Gershenson D and Mutch DG: Pelvic irradiation with concurrent chemotherapy versus pelvic and para-aortic irradiation for high-risk cervical cancer: an update of radiation therapy oncology group trial (RTOG) 90-01. J Clin Oncol 22: 872-880, 2004.

7. Green JA, Kirwan JM, Tierney JF, Symonds P, Fresco L, Colingwood $M$ and Williams CJ: Survival and recurrence after concomitant chemotherapy and radiotherapy for cancer of the uterine cervix: a systematic review and meta-analysis. Lancet 358: 781-786, 2001 .

8. Kim YB, Cho JH, Keum KC, Lee CG, Seong J, Suh CO and Kim GE: Concurrent chemoradiotherapy followed by adjuvant chemotherapy in uterine cervical cancer patients with high-risk factors. Gynecol Oncol 104: 58-63, 2007.

9. Choi CH, Lee JW, Kim WY, Nam HR, Kim BG, Hum SJ, Lee JH and Bae DS: Phase II study of consolidation chemotherapy after concurrent chemoradiation in cervical cancer: preliminary results. Int J Rad Oncol Biol Phys 68: 817-822, 2007.

10. Japan Society of Gynecologic Oncology: Cervical cancer treatment guidelines, 2007.

11. Ikushima H, Osaki K, Furutani S, Yamashita K, Kawanaka T, Kishida Y, Wamoto S, Takegawa Y, Kudoh T and Nishitani H: Chemoradiation therapy for cervical cancer: toxicity of concurrent weekly cisplatin. Rad Med 24: 115-121, 2006.

12. Sakuragi N: Up-to-date management of lymph node metastasis and the role of tailred lymphadenenctomy in cervical cancer. Int J Clin Oncol 12: 165-175, 2007.

13. Berman ML, Keys H, Creasman W, Reasman W, DiSaia P, Bundy B and Blessing J: Survival and patterns of recurrence in cervical cancer metastatic to periaortic lymph nodes. Gynecol Oncol 19: 8-16, 1984.

14. Benedetti PP, Basile S and Angioli R: Pelvic and aortic lymphadenectomy in cervical cancer: the standardization of surgical procedure and its clinical impact. Gynecol Oncol 113: 284-290, 2009.

15. Vidaurreta J, Bermudez A, Di Paola G and Sardi J: Laparoscopic staging in locally advanced cervical carcinoma: a new possible philosophy? Gynecol Oncol 75: 366-371, 1999.

16. Grigsby PW, Dehdashi F and Siegel BA: FDG-PET evaluation of carcinoma of the cervix. Clin Pos Image 2: 105-109, 1999.

17. Rose PG and Bundy BN: Chemoradiation for locally advanced cervical cancer: does it help? J Clin Oncol 20: 891-893, 2002.

18. Grigsby PW, Perez CA, Chao KS, Herog T, Mutch DG and Rader J: Radiation therapy for carcinoma of the cervix with biopsy-proven positive para-aortic lymph nodes. Int J Rad Oncol Biol Phys 49: 733-738, 2001.

19. Walker JL, Morrison A, DiSilvestro P and von Gruenige VE: A phase I/II study of extended field radiation therapy with concomitant paclitaxel and cisplatin chemotherapy in patients with cervical carcinoma metastatic to the para-aortic lymph nodes. Gynecol Oncol 112: 78-84, 2009.

20. Zhang MQ, Liu SP and Wang XE: Concurrent chemotherapy with paclitaxel and nedaplatin followed by consolidation chemotherapy in locally advanced squamous cell carcinoma of the uterine cervix: preliminary results of phase II study. Int Rad Oncol Biol Phys 78: 821-827, 2010.

21. Rose PG, Lessing JA, Gershenson DM and McGehee R: Paclitaxel and cisplatin as first-line therapy in recurrent or advanced squamous cell carcinoma of the cervix: a gynecologic oncology group study. J Clin Oncol 17: 2676-2680, 1999.

22. Sit AS, Kelley JL, Gallion HH, Kunschner AJ and Edwards RP: Paclitaxel and carboplatin for recurrence or persistent cancer of the cervix. Cancer Invest 22: 368-373, 2004. 